\title{
Dynamic vortex arrays created by starfish larvae
}

\author{
William Gilpin, ${ }^{1}$ Vivek N. Prakash,${ }^{2}$ and Manu Prakash ${ }^{2, *}$ \\ ${ }^{1}$ Department of Applied Physics, Stanford University, Stanford, California 94305, USA \\ ${ }^{2}$ Department of Bioengineering, Stanford University, Stanford, California 94305, USA
}

(Received 10 August 2017; published 29 September 2017)

\begin{abstract}
This paper is associated with a video winner of a 2016 APS/DFD Milton van Dyke Award for work presented at the DFD Gallery of Fluid Motion. The original video is available from the Gallery of Fluid Motion, https://doi.org/10.1103/APS.DFD.2016.GFM.V0055
\end{abstract}

DOI: 10.1103/PhysRevFluids.2.090501

The first animals evolved at low Reynolds numbers, and so understanding how they adapted to their viscous surroundings may shed light on the general mechanisms by which environmental forces shape evolutionary processes.

We spawned the larvae of Patiria miniata, a common starfish typically used as a model organism for developmental biology, and we observed that the larvae produce vortices around their bodies that form elegant arrays of eddies when the larva is immobilized on a microscope slide (Fig. 1). Unlike adult starfish, larval starfish are free swimming for most of their first two months of development. In our recent work [1], we found that these vortex structures work in tandem with swimming to
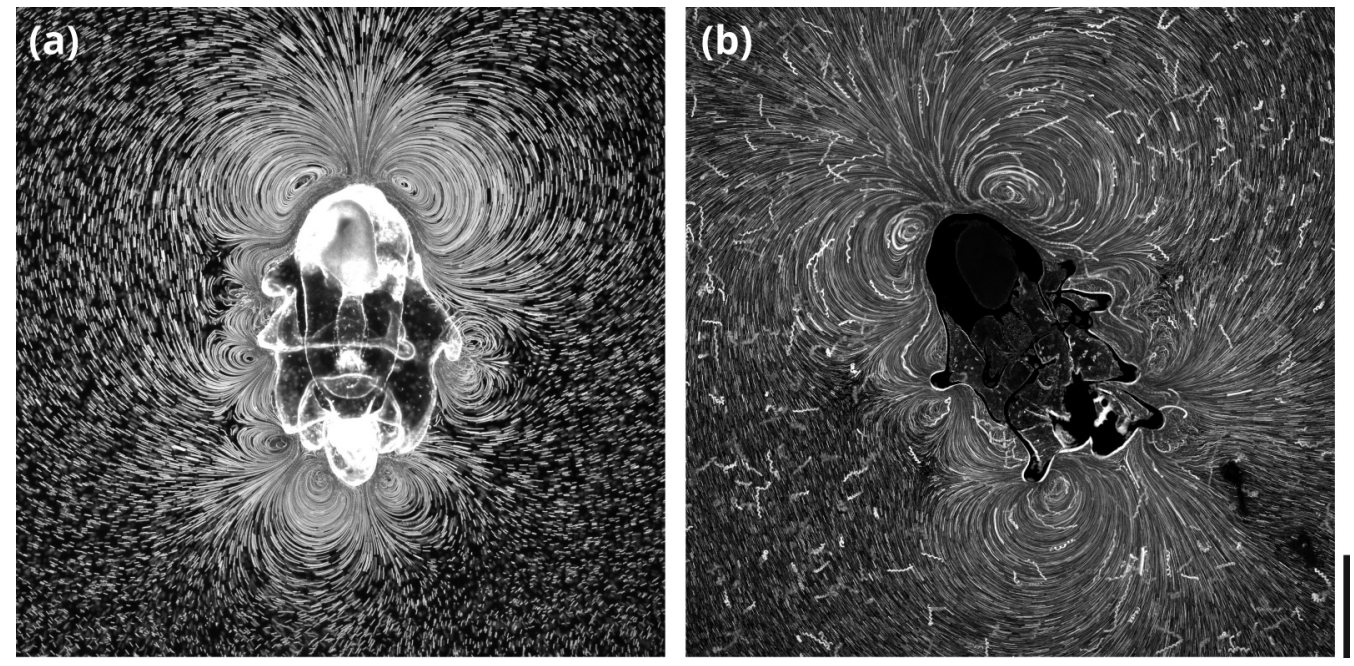

FIG. 1. (a) A 3-s time-lapse image of the currents around the larva of the California bat star, Patiria miniata. Flows are visualized by adding $6-\mu \mathrm{m}$ beads to the water and computationally creating the maximum-intensity projection of a movie of the beads circumscribing the flow field [1]. (b) The role of the flow field in feeding is visualized by adding $0.75-\mu \mathrm{m}$ beads (thinner streaks) as well as $\sim 5.5 \mu \mathrm{m}$ algae on which the larva feeds (thicker, helical trajectories). Scale bar: $500 \mu \mathrm{m}$. The video associated with these images is available at https://doi.org/10.1103/APS.DFD.2016.GFM.V0055

*Corresponding author: manup@stanford.edu

Published by the American Physical Society under the terms of the Creative Commons Attribution 4.0 International license. Further distribution of this work must maintain attribution to the author(s) and the published article's title, journal citation, and DOI. 

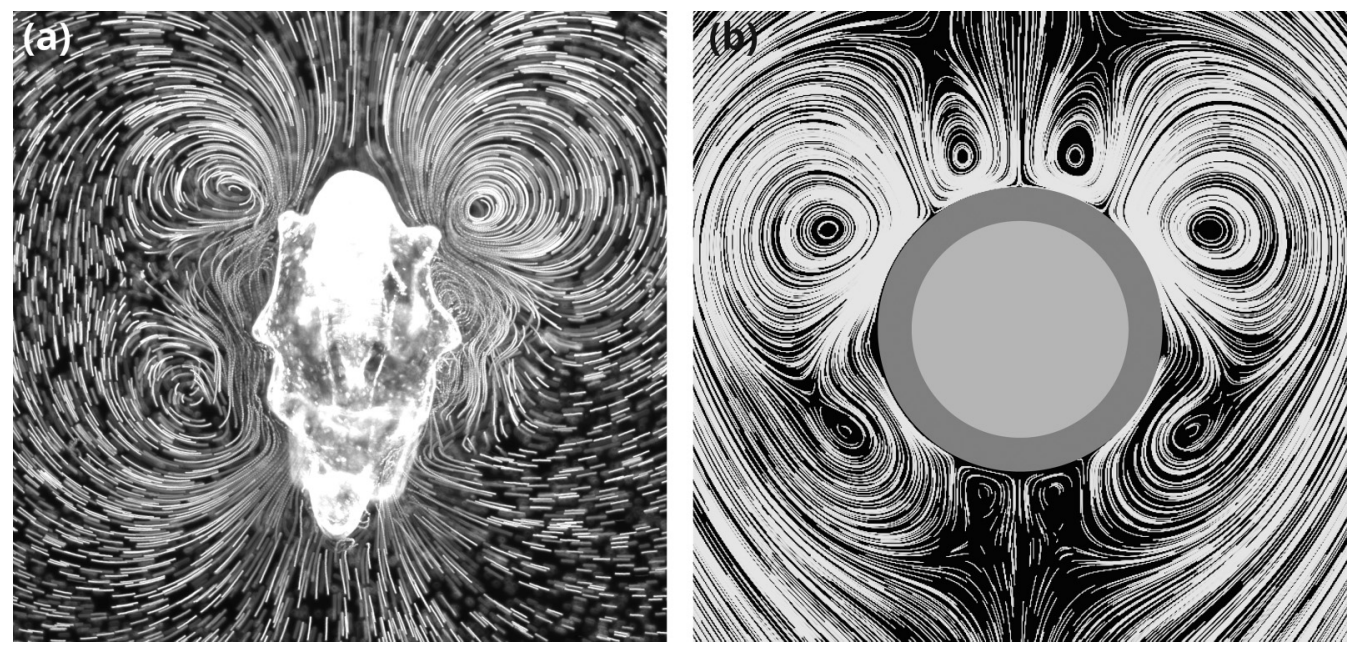

FIG. 2. (a) A feeding current produced by a starfish larva and (b) the streamlines produced by a best-fit "squirmer" model of the flow field. A stokeslet has been subtracted from the computed squirmer model flow, in order to mimic the effects of immobilization [1]. The video associated with these images is available at https://doi.org/10.1103/APS.DFD.2016.GFM.V0055

enhance each larva's ability to intercept and ingest algae, their primary food source. Immobilization of the swimmer combined with computational reconstruction of the pathlines of tracer particles [2,3] allows straightforward visualization of the elegant and dynamic array of vortices that forms around the body.

Like many swimmers at low Reynolds numbers, starfish larvae use beating cilia as their primary means of propulsion. However, unlike many canonical Stokesian swimmers like bacteria or phytoplankton [4-6], each starfish larva has tens of thousands of such cilia, all controlled by the larva's central nervous system. For this reason, we can model the flow field as that produced by a continuous, active "slip" boundary condition on the surface of the larva's body-allowing us to study mathematically the properties of the flow using the well-known "squirmer" model of propulsion at low Reynolds number (Fig. 2) [7]. This mathematical model provides insight into the precise adaptive benefit of creating such intricate fluidic structures by suggesting a potential tradeoff between the larvae's ability to swim for dispersal and its ability to efficiently capture food in the nonuniform oceanic environment [8].

Intense competition for survival and perpetually changing environmental conditions have conspired to produce an endless diversity of shapes and forms of life in the ocean. Fluid dynamics holds the promise of unearthing general organizing principles underlying the many forms that life takes underwater by connecting the physical fluid forces that act on living things with the fleeting evolutionary forces that shape their adaptation.

W.G. thanks the National Geographic Society for support through the Young Explorers Grant program, as well as the U.S. Department of Defense for support through the NDSEG Fellowship program. This work was supported by ARO MURI Grant No. W911NF-15-1-0358 and an NSF CAREER Award (to M.P.).

[1] W. Gilpin, V. N. Prakash, and M. Prakash, Vortex arrays and ciliary tangles underlie the feeding-swimming trade-off in starfish larvae, Nat. Phys. 13, 380 (2017). 


\section{DYNAMIC VORTEX ARRAYS CREATED BY STARFISH LARVAE}

[2] W. Gilpin, V. N. Prakash, and M. Prakash, Flowtrace: Simple visualization of coherent structures in biological fluid flows, J. Exp. Biol. (2017), doi:10.1242/jeb.162511.

[3] W. Gilpin, V. N. Prakash, and M. Prakash, Reply to 'Boundary effects on currents around ciliated larvae,' Nat. Phys. 13, 521 (2017).

[4] J. S. Guasto, R. Rusconi, and R. Stocker, Fluid mechanics of planktonic microorganisms, Annu. Rev. Fluid Mech. 44, 373 (2012).

[5] J. B. Kirkegaard, A. O. Marron, and R. E. Goldstein, Motility of Colonial Choanoflagellates and the Statistics of Aggregate Random Walkers, Phys. Rev. Lett. 116, 038102 (2016).

[6] A. Mathijssen, R. Jeanneret, and M. Polin, Universal entrainment mechanism governs contact times with motile cells, arXiv:1704.05264.

[7] J. Blake, Self-propulsion due to oscillations on the surface of a cylinder at low Reynolds number, Bull. Aust. Math. Soc. 5, 255 (1971).

[8] V. I. Fernandez and R. Stocker, Hydrodynamics: Modus vivendi, Nat. Phys. 13, 326 (2017). 